\title{
Aplicación de Elastografía por Retorno Temporal a la evaluación de textura en quesos*
}

\author{
Brum, J. (1), Barrios S. (2), Ares, G. (2), Lema, P. (2), Canetti, R. (3), Negreira, C. (1) \\ Contacto: sbarrios@fing.edu.uy \\ (1) Laboratorio de Acústica Ultrasonora. Instituto de Física, Facultad de Ciencias - ${ }^{(2)}$ Instituto de Ingeniería Química - \\ (3) Instituto de Ingeniería Eléctrica, Facultad de Ingeniería. Universidad de la República, Uruguay. \\ Recibido: 13/11/2009 - Aprobado: 7/12/2009
}

*Primer premio de INNOVA 2009: IV Simposio Internacional de Innovación y Desarrollo de Alimentos, (Montevideo 30 de setiembre, 1-2 de octubre de 2009). Montevideo: LATU, 2009.

\begin{abstract}
$\underline{\text { Resumen }}$
EI objetivo de este trabajo fue estudiar la aplicación de la técnica acústica Elastografía por Retorno Temporal (TRE) a la evaluación de la textura de distintos tipos de queso. Se evaluaron ocho muestras comerciales de diferente textura mediante las técnicas de TRE y Análisis de Perfil de Textura (TPA). Ambas metodologías permitieron detectar diferencias en la textura de las muestras, aportando resultados concordantes. De acuerdo a la regresión de mínimos cuadrados parciales realizada (PLS), el ancho de focalización obtenido por TRE se correlacionó positivamente $\left(R^{2}=0.89\right)$ con los parámetros dureza y rigidez del TPA. Esto indica que la técnica de TRE sería un método adecuado para evaluar la textura de los quesos de forma no destructiva. Palabras clave: Técnicas acústicas, parámetros elásticos, evaluación no destructiva.
\end{abstract}

\begin{abstract}
The aim of this work was to study the application of the acoustic technique Time Reversal Elastography (TRE) for texture evaluation of different types of cheese. Eight commercial cheese samples were evaluated using TRE and Texture Profile Analysis (TPA). Both methodologies were able to identify differences in the texture of the evaluated cheese samples, providing similar results. According to the partial least squares regression performed, the focalization width determined by TRE was positively correlated $\left(R^{2}=0.89\right)$ to hardness and stiffness. This indicates that TRE could be considered as an adequate method for the non destructive evaluation of cheese texture.

Keywords: Acoustic techniques, elastic parameters, non destructive evaluation.
\end{abstract}

\section{Introducción}

La aplicación de técnicas de monitoreo y control de calidad que permitan la evaluación de propiedades de alimentos de manera rápida y no destructiva es de gran interés para la industria alimentaria. En los últimos años se ha verificado el gran potencial que poseen las técnicas acústicas para la evaluación de distintas propiedades en alimentos como queso, carne, frutas y aceites (Benedito et al., 2002; Benedito et al., 2006a). La aplicación de estas técnicas constituye una alternativa no destructiva y fácilmente automatizable. En particular, la aplicación de técnicas acústicas permite la evaluación de la textura de diferentes tipos de queso (Benedito et al., 2006b).

La velocidad de propagación del ultrasonido se utilizó como parámetro ultrasónico para el seguimiento de los cambios de textura que se dan a lo largo de la maduración en quesos Manchego, Cheddar y Mahon (Benedito et al., 2000a, 2000b, 2006a). Los resultados de estos trabajos muestran que la medida de textura por ultrasonido es posible, verificándose la correlación entre la velocidad de ultrasonido y parámetros de textura tales como dureza y rigidez. Sin embargo, se verificó una influencia importante de la atenuación en las medidas de ultrasonido realizadas sobre hormas de queso entero, por lo cual las medidas sólo resultaban adecuadas en muestras de $2 \mathrm{~cm}$ de largo (Benedito et al., 2006b). Esto convierte al ensayo en destructivo, debiéndose buscar alternativas que permitan la evaluación de muestras de queso sin cortar

Benedito et al. (2006b) evaluaron la aplicación de una técnica de pulso - respuesta que consistió en golpear una horma de queso Manchego y evaluar la respuesta a dicha excitación a lo largo del proceso de maduración. La técnica resultó adecuada para la evaluación de los cambios en la textura de la superficie del queso, pero no dio buenos resultados en la parte interna del queso.

La técnica de Elastografía por Retorno Temporal (TRE) se ha desarrollado en los últimos tres años en el Laboratorio de Acústica Ultrasonora del Instituto de Física, Facultad de Ciencias, Universidad de la República (Brum et al., 2008; Catheline et al., 2008; Benech et al., 2009). La técnica de TRE fue concebida para la evaluación de las propiedades mecánicas de tejidos biológicos y ha sido utilizada en la evaluación de terneza en carne, control de temperatura en músculo esquelético y evaluación de textura en quesos. Consiste en la generación de una señal acústica mediante una excitación externa. Una vez adquirida esta señal, se reconstruye numéricamente el proceso de focalización espacio-temporal (Brum et al., 2008). El ancho a -6dB del pico de la focalización espacial en la reconstrucción del proceso de focalización permite evaluar la longitud de onda del pulso. La misma está relacionada con la elasticidad del medio, es decir, el ancho de focalización varía en función de su textura. Una ventaja que presenta esta técnica es que puede ser aplicada a una horma entera de queso sin necesidad de cortar muestras, por lo cual podría ser aplicada a la evaluación no destructiva de la textura de quesos. Además, es una técnica de bajo costo y fácil implementación.

El objetivo de este trabajo fue estudiar la aplicación de la técnica acústica de baja frecuencia Elastografía por Retorno Temporal (TRE) a la evaluación de la textura de distintos tipos de queso.

\section{Materiales y Métodos}

\section{Muestras}

Se evaluaron ocho muestras comerciales de queso de distintas características de textura: cuatro muestras de Cuartirolo, dos de 
Colonia, un Danbo y un Magro. Las muestras, provenientes de empresas lácteas de los departamentos de Colonia y Montevideo, fueron adquiridas en supermercados de la ciudad de Montevideo y transportadas en empaques aislados térmicamente hasta el laboratorio donde se realizaron los ensayos.

\section{Elastografía por Retorno Temporal}

El montaje experimental para las medidas de TRE se muestra en la Figura 1. El dispositivo consta de un martillo de punta de goma montado sobre un eje giratorio que golpea el queso de forma controlada. El golpe del martillo constituye la excitación externa del queso. La adquisición de la respuesta acústica de la muestra de queso a la excitación externa se logró a través de un arreglo de ocho sensores pasivos (Murata PKS1-4A) con un ancho de banda desde los $0.1 \mathrm{~Hz}$ hasta los $5 \mathrm{kHz}$. Este arreglo puede ser colocado en cualquier lugar sobre la superficie del queso. La señal acústica de los ocho sensores se digitalizó con una tarjeta adquisidora de ocho canales, National Instruments NI USB-6009, a una frecuencia de muestreo de $5 \mathrm{kHz}$. Utilizando la técnica de filtro inverso (Tanter et al., 2001), se trataron las señales acústicas para la recreación del proceso de refocalización temporal. Por medio de este proceso se obtuvo el ancho del pico a $-6 \mathrm{~dB}$ de la focalización espacial (ancho de focalización o amplitud).

\section{Análisis de Perfil de Textura}

Se realizó un Análisis de Perfil de Textura utilizando un Analizador de Textura TA.XT2i (Stable Micro Systems, Godalming, UK) Para cada tipo de queso analizado se realizaron 10 ensayos. El ensayo consistió en la doble compresión de cubos de queso de $2 \mathrm{~cm}$ de arista. Las condiciones de ensayo fueron las siguientes: velocidad de pre-ensayo: $1 \mathrm{~mm} / \mathrm{s}$; velocidad de ensayo: $0,5 \mathrm{~mm} / \mathrm{s}$; velocidad de post-ensayo: $1 \mathrm{~mm} / \mathrm{s}$; porcentaje de compresión: $50 \%$ (Benedito et al., 2006a). Utilizando los datos adquiridos mediante el software Texture Expert Exceed Version 1.0 (Stable Micro Systems, Godalming, UK), se calcularon para cada tipo de queso los parámetros dureza, rigidez, elasticidad y cohesividad, según Fox et al. (2000).

La dureza fue calculada como la fuerza máxima durante la primera compresión, la rigidez como el área bajo la curva de fuerza vs tiempo durante la primera compresión. La cohesividad se calculó como el cociente entre el área bajo la curva durante la segunda compresión y el área bajo la curva durante la primera compresión; mientras que la elasticidad se calculó como el cociente entre el tiempo necesario para alcanzar el máximo de fuerza durante la segunda compresión y el tiempo necesario para alcanzar el máximo de fuerza durante la primera compresión.

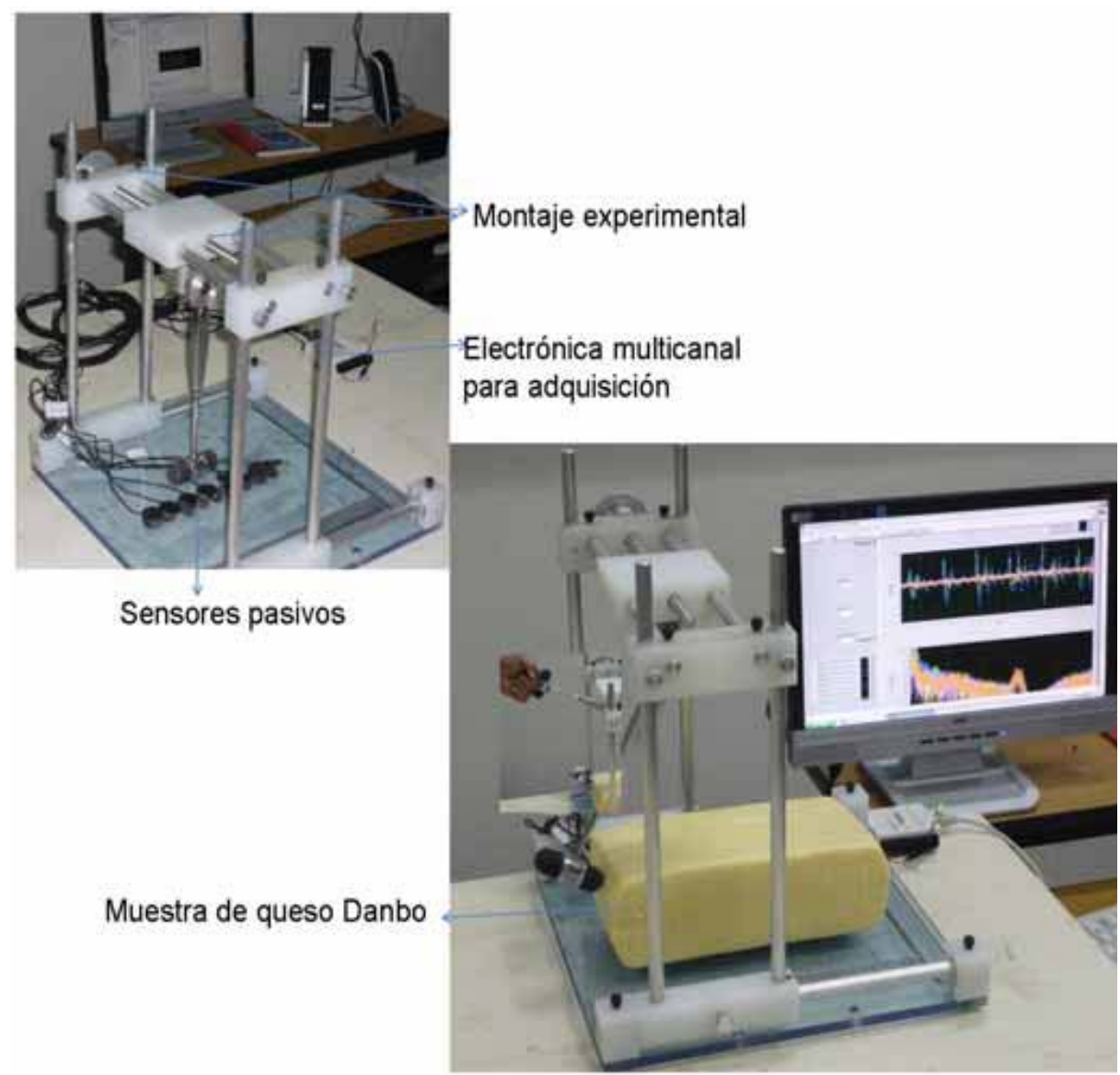

Figura 1. Montaje experimental para la experiencia de TRE. 


\section{Análisis de datos}

\section{- Análisis de varianza (ANOVA)}

Se realizó un ANOVA con los parámetros de textura instrumental medidos por TPA, considerando el tipo de queso como variable independiente. Los análisis se realizaron utilizando el software estadístico Genstat Discovery Edition 2 (VSN International, Oxford, UK), calculando diferencias significativas por medio del test de Tukey $(\alpha=0.05)$.

\section{- Regresión de mínimos cuadrados parciales (PLS)}

Se realizó un PLS considerando el ancho de focalización como variable dependiente y los parámetros de textura instrumental como variables independientes. Este análisis busca explicar las variaciones en las medidas acústicas a través de las variaciones en las medidas de textura instrumental de los quesos. La técnica de regresión PLS es sumamente útil cuando se trabaja con un alto número de variables, las cuales pueden estar interrelacionadas (Martens \& Martens, 1986). La regresión PLS se basa en encontrar un modelo de regresión lineal mediante la proyección de las variables dependientes e independientes consideradas en un nuevo espacio. Este nuevo espacio es creado considerando nuevas variables denominadas factores del PLS, los cuales son generados utilizando combinaciones lineales de las variables dependientes e independientes consideradas en el análisis.

Todos los análisis estadísticos se realizaron utilizando el software XL-Stat 2009 (Addinsoft, Inc., Brooklyn, NY, USA).

\section{Resultados y Discusión}

Los resultados de la técnica de TRE fueron comparados con los de la técnica de TPA para estudiar la aplicabilidad TRE a la evaluación de la textura de quesos. En primer lugar, se verificó que los quesos evaluados presentaran diferencias significativas en los parámetros de textura instrumental. Una vez confirmadas estas diferencias, se buscó una correlación entre los parámetros de textura instrumental y el ancho de focalización medido por TRE, utilizando el análisis estadístico de mínimos cuadrados parciales (PLS).

ANOVA. El ANOVA mostró que existen diferencias significativas $(p<0.05)$ entre los distintos tipos de queso para los parámetros de textura instrumental, como se muestra en la Tabla 1. Estos resultados indican que los quesos evaluados presentaron diferencias significativas en sus características de textura.

\begin{tabular}{|l|c|c|c|c|}
\hline Muestra & Dureza (N) & Rigidez (N.s) & Elasticidad & Cohesividad \\
\hline 1 & $5.9^{\mathrm{a}}$ & $45.5^{\mathrm{a}}$ & $0.57^{\mathrm{a}}$ & $0.43 \mathrm{a}$ \\
\hline 2 & $7.2^{\mathrm{b}}$ & $54.2^{\mathrm{b}}$ & $0.60^{\mathrm{b}}$ & $0.50^{\mathrm{b}}$ \\
\hline 3 & $20.9^{\mathrm{c}}$ & $168.7^{\mathrm{c}}$ & $0.66^{\mathrm{c}}$ & $0.52^{\mathrm{b}}$ \\
\hline 4 & $22.3^{\mathrm{d}}$ & $187.8^{\mathrm{d}}$ & $0.74^{\mathrm{d}}$ & $0.69^{\mathrm{d}}$ \\
\hline 5 & $35.9^{\mathrm{c}}$ & $270.6^{\mathrm{c}}$ & $0.76^{\mathrm{c}}$ & $0.79^{\mathrm{f}}$ \\
\hline 6 & $38.6^{\mathrm{f}}$ & $283.4^{\mathrm{r}}$ & $0.77^{\mathrm{c}}$ & $0.58^{\mathrm{c}}$ \\
\hline 7 & $44.7^{\mathrm{g}}$ & $318.4^{\mathrm{g}}$ & $0.81^{\mathrm{f}}$ & $0.8^{\mathrm{f}}$ \\
\hline 8 & $46.3^{\mathrm{b}}$ & $343.9^{\mathrm{h}}$ & $0.77^{\mathrm{c}}$ & $0.77^{\mathrm{c}}$ \\
\hline
\end{tabular}

Tabla 1. Valores promedio de los parámetros de textura instrumental determinados para los ocho quesos evaluados.

Nota: Valores promedio dentro de una misma columna indican diferencias significativas de acuerdo al test de Tukey $(p<0.05)$.

PLS. Se realizó un PLS para estudiar la relación existente entre el ancho de focalización obtenido por TRE y los parámetros de textura determinados en el TPA. Los dos primeros factores del PLS explicaron el 98,6\% de la varianza de los datos de textura instrumental (variables independientes: X) y el 89,1\% de la varianza de los datos acústicos (variable dependiente: Y). Estos resultados indican que las variables de textura instrumental y el ancho de focalización pueden representarse en el espacio determinado por los dos factores del PLS, sin perder una cantidad importante de información.

En el Gráfico 1 se representan las variables en los dos primeros factores del PLS. En éste, dos variables perfectamente correlacionadas entre sí estarán representadas por vectores con la misma dirección, mientras que dos variables que no estén correlacionadas estarán representadas por vectores perpendiculares. Como se observa en el Gráfico 1, los cuatro parámetros de textura instrumental estuvieron fuertemente correlacionados entre sí. En particular, la dureza y la rigidez de los quesos fueron los que presentaron la mayor correlación.

Además, se observa que el ancho de focalización se correlaciona positivamente en mayor medida con la dureza y la rigidez de las muestras. Este resultado sugiere que el ancho de focalización depende fuertemente de la textura de los quesos, en particular de los parámetros dureza y rigidez determinados a través del TPA. Debido a la existencia de una correlación positiva entre el ancho de focalización y la dureza y rigidez determinadas en el TPA, puede concluirse que el ancho de focalización determinado por TRE aumentó al aumentar la dureza y la rigidez de las muestras.

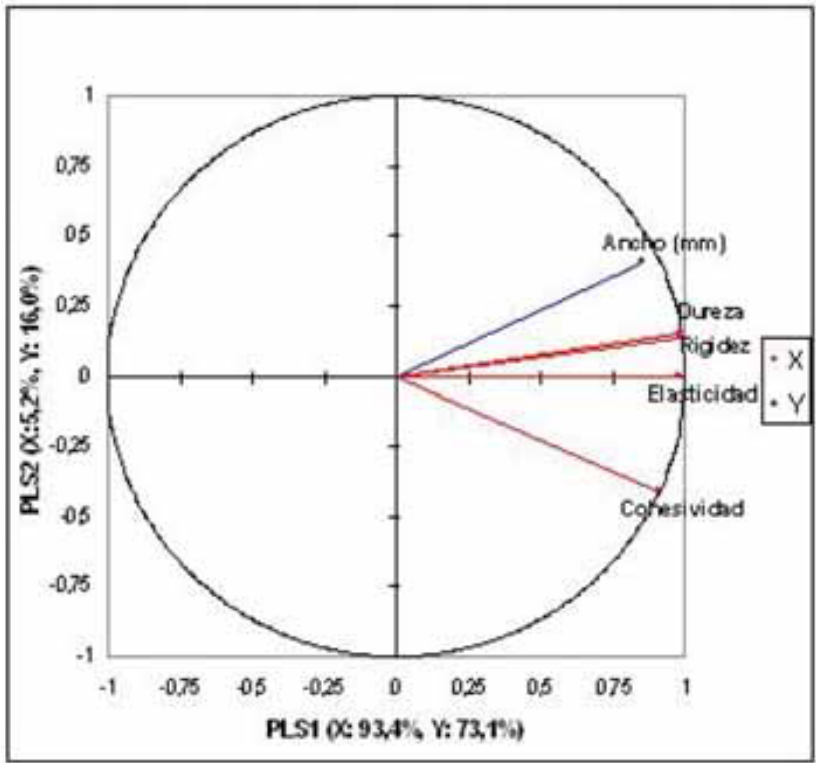

Gráfico 1. Representación de las variables de textura instrumental y acústicas de las muestras de queso evaluadas en los dos primeros factores del PLS.

La regresión PLS permitió obtener una ecuación con la cual puede predecirse el ancho de focalización a partir de una combinación lineal de parámetros de textura instrumental. La ecuación que se obtiene, que explica el $89,1 \%$ de la variación de los datos, es la siguiente:

Ancho de focalización $(\mathrm{mm})=26,4+0,176 *$ Dureza + 0,023*Rigidez $+16,67 *$ Elasticidad $-19,98 *$ Cohesividad

Los coeficientes de la ecuación con sus correspondientes intervalos de confianza se presentan en el Gráfico 2, donde se puede apreciar que la dureza y rigidez presentaron coeficientes significativamente positivos, lo cual indica que influyeron en los valores de ancho de focalización obtenidos. Es decir, este parámetro acústico dependió principalmente de estos dos parámetros de textura de los quesos. Sin embargo, la elasticidad y cohesividad no presentaron coeficientes significativos, lo que sugiere que no afectaron significativamente las medidas acústicas. Por lo tanto el ancho de focalización estuvo principalmente relacionado con la dureza y rigidez de los quesos. 


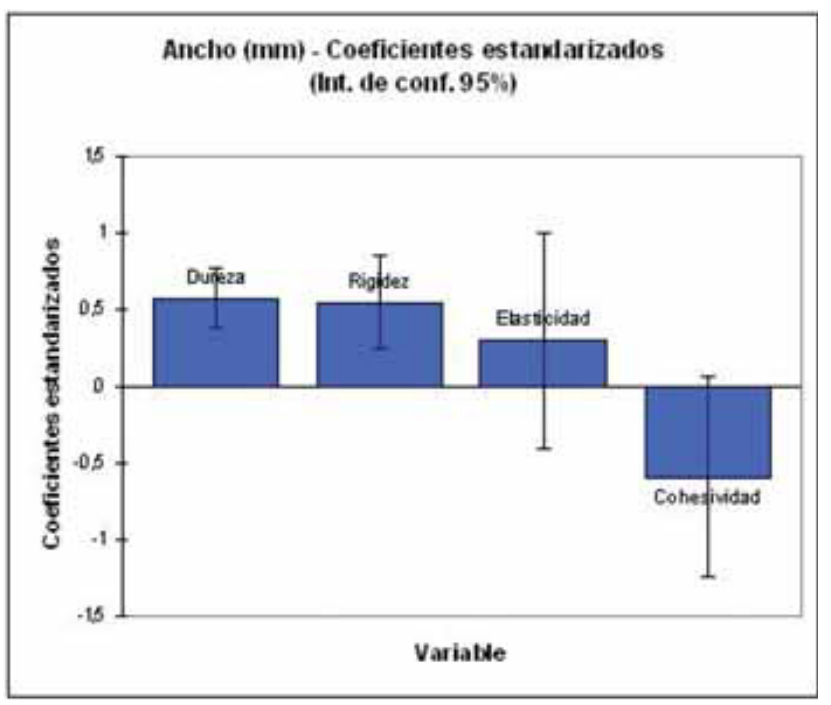

Gráfico 2. Coeficientes estandarizados en la regresión PLS de ancho de focalización en función de los valores de textura instrumental.

Considerando únicamente los parámetros que presentaron coeficientes significativos el modelo se reduce a la siguiente expresión:

\section{Ancho de focalización $(\mathrm{mm})=26,4+0,176 *$ Dureza + $0,023 *$ Rigidez}

Como se expresa en el Gráfico 3, los anchos predichos por el modelo fueron muy cercanos a los determinados experimentalmente para todos los quesos evaluados. Esto hace posible concluir que el ancho de focalización determinado por TRE permitió predecir la dureza y rigidez de las muestras de queso evaluadas.

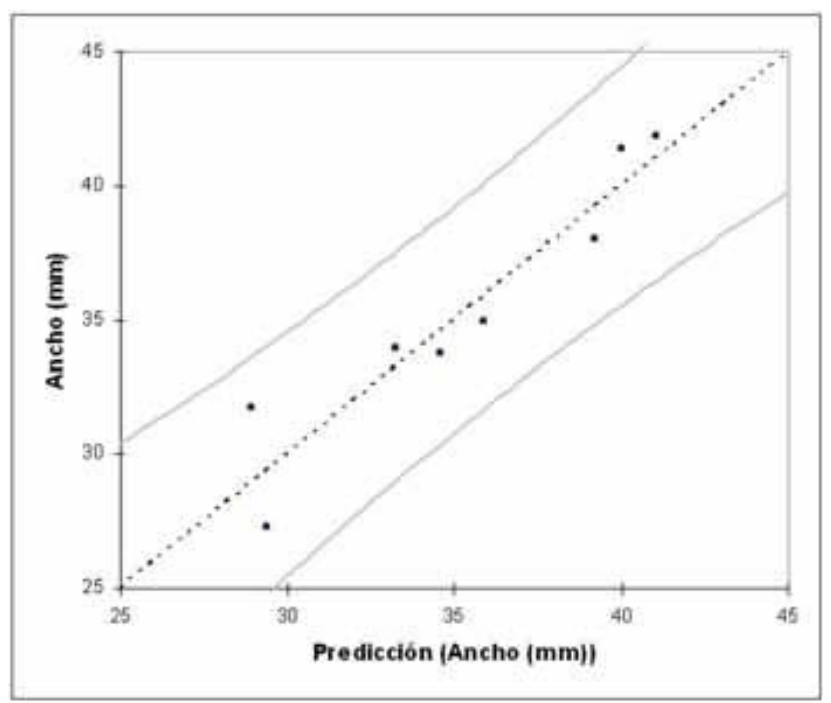

Gráfico 3. Valores de ancho de focalización de las ocho muestras de queso evaluadas en función de los valores predichos a partir de la regresión PLS de los valores de textura instrumental.

\section{Conclusiones}

El método TRE permitió detectar diferencias en la textura de los quesos, reflejando principalmente sus características de durezay rigidez. Esta técnica posee la ventaja de ser no destructiva, contrariamente a las técnicas que son utilizadas actualmente. Los resultados de este trabajo indican que dicho método resultaría adecuado para evaluar la textura de quesos de forma no destructiva. Se deberían medir otros tipos de quesos por el método TRE para poder confirmar la aplicabilidad de la técnica.

\section{Reconocimientos}

La realización de estos ensayos fue posible gracias al apoyo del Ministerio de Educación y Cultura, a través del proyecto del Programa de Desarrollo Tecnológico PDT 34/09.

\section{Referencias}

- BENECH, N.; CATHELINE, S.; BRUM, J.; GALLOT, T.; NEGREIRA, C. 1-D Elasticity assesment in soft solids from shear wave correlation: the time reversal approach. En: IEEE Transactions on Ultrasonics. Ferroelectrics and Frequency Control. 2009, 59(11):2400-2410.

- BENEDITO, J.; CÁRCEL, J.A.; CLEMENTE, G.; MULET, A. Cheese maturity assessment using ultrasonics. En: Journal of Diary Science. 2000a, (83):248-254.

- BENEDITO, J.; CÁRCEL, J.A.; SANJUAN, N.; MULET, A. Use of ultrasound to assess cheeese charachteristics.

En: Ultrasonics. 2000b, (38):727-730.

- BENEDITO, J.; CÁRCEL, J.A.; GONZALEZ, R.; MULET, A. Application of low intensity ultrasonics to cheese manufacturing processes. En: Ultrasonics. 2002, (40):19-23.

- BENEDITO, J.; SIMAL, S.; CLEMENTE, G.; MULET, A. Manchego cheese texture evaluation by ultrasonics and surface probes. En: International Dairy Journal. 2006a, (16):431-438.

- BENEDITO, J.; CONDE, T.; CLEMENTE, G.; MULET, A. Use of the acoustic impulse - response technique for the nondestructive assessment of Manchego cheese texture. En: Journal of Dairy Science. 2006b, (89):4490-4502.

- BRUM, J.; CATHELINE, S.; BENECH, N.; NEGREIRA, C. Shear elasticity estimation from surface wave: the time reversal approach. En: Journal of the Acoustical Society of America. 2008, 124(6):3377-3380.

- CATHELINE, S.; BENECH, N.; BRUM, J.; NEGREIRA, C. Time reversal of elastic waves in soft solids. En: Physical Review Letters. 2008, (100):064301-1-064301-4.

- FOX, P.F.; GUINEE, T.P.; COGAN, T.M.; MCSWEENEY, P.L.H. Fundamentals of cheese science. Gaithersburg: Aspen Publishers, 2000.

- MARTENS, M.; MARTENS, H. Partial least squares regression. En: PIGGOTT, J. R. Statistical procedures in food research. vol. 9. London: Elsevier Applied Science, 1986. pp. 293-359.

- TANTER, M.; AUBRY, J.F.; GERBER, J.; GERBER, J.; THOMAS, L.; FINK, M. Optimal focusing by spatio-temporal inverse filter I. Basic principles. En: Journal of the Acoustical Society of America. 2001, 110(1):37-43. 